\title{
A insuficiência da valva aórtica na dissecção crônica da aorta proximal: troca ou reconstrução valvar?
}

\author{
Ricardo BEYRUTI*, Noedir A. G. STOLF ${ }^{\star \star}$, Luiz Felipe P. MOREIRA ${ }^{\star *}$, Paulo M. PÊGO-FERNANDES ${ }^{\star *}$, \\ Charles MADY**, Adib D. JATENE ${ }^{\star *}$
}

RBCCV 44205-205

\begin{abstract}
BEYRUTI, R.; STOLF, N. A. G.; MOREIRA, L. E. P.; PÊGO-FERNANDES, P. M.; MADY, C.; JATENE, A. D. - A insuficiência da valva aórtica na dissecção crônica da aorta proximal: troca ou reconstrução valvar? Rev. Bras. Cir. Cardiovasc., 8 (2):108-117, 1993.

RESUMO: Com o objetivo de avaliar os resultados clínicos e ecocardiográficos tardios obtidos com a correção da insuficiência aórtica decorrente da dissecção crônica da aorta proximal, foram estudados 48 pacientes consecutivos operados entre janeiro de 1980 e dezembro de 1989, separados em 2 grupos de 24 pacientes cada. Grupo A - pacientes nos quais a valva aórtica foi preservada pela "resuspensão comissural"; Grupo B - pacientes nos quais a valva aórtica foi substituida. Na avaliação ecocardiográfica pré-operatória, os pacientes do Grupo $\mathrm{B}$ apresentavam grau de insuficiência aórtica, diâmetros ventriculares (sistólico e diastólico) e da aorta ascendente significativamente maiores do que os do Grupo $\mathbf{A}(\mathrm{p}=0,03)$, sendo comparáveis nos demais parâmetros. A mortalidade hospitalar foi $12,5 \%$ no Grupo $A$ e de $4,17 \%$ no Grupo B e a sobrevida aos 7 anos, respectivamente, $75,75 \% \pm 9,82 \%$ e $82,72 \% \pm 7,87 \%$ (NS). A avaliação clínica mostrou que, no pós-operatório, houve melhora significativa $(p<0,001)$ e semelhante dos parâmetros nos dois grupos. A comparação ecocardiográfica pré e pós-operatória tardia mostrou, da mesma forma, redução importante dos diâmetros sistólico e diastólico do ventrículo esquerdo e no diâmetro da aorta $(p<0,05)$, mantendo-se inalteradas as fraçōes de encurtamento e de ejeção ventriculares nos pacientes dos dois grupos. Nos pacientes do Grupo A, entretanto, houve persistência de insuficiência aórtica residual $(p=0,03)$. Os autores concluem que, com as duas técnicas empregadas, o tratamento cirúrgico da dissecção da aorta ascendente com insuficiência aórtica associada permite sobrevida imediata e tardia satisfatórias e nítida melhora funcional. Nos pacientes do Grupo A, a insuficiência aórtica residual detectada à ecocardiografia não produziu sintomas ou repercussão hemodinâmica tardios e, desta forma, preconizam a preservação da valva, sempre que tecnicamente possível.
\end{abstract}

DESCRITORES: valva aórtica, cirurgia; valvas cardiacas, cirurgia.

\section{INTRODUÇÃO}

Dentre as afecções que acometem a aorta, poucas são as que ocorrem de forma tão dramática e com manifestações clínicas tão variadas como a dissecção aórtica. Em suas formas mais graves, o tratamento operatório constitui-se na única modalidade terapêutica que pode alterar favoravelmente a evolução natural da doença. A indicação cirúrgica e o sucesso do tratamento dependem não somente da presença e magnitude de complicações inerentes, mas também do segmento vascular comprometido e da oportunidade de sua realização.

Face à grande variabilidade clínica e dos resultados obtidos com diferentes técnicas para sua correção, muitos autores têm considerado que as afeç̧ões da aorta devem ser estudadas e trata-

\footnotetext{
Trabalho realizado no Instituto do Coração do Hospital das Clínicas da Faculdade de Medicina da Universidade de São Paulo. Apresentando ao $20^{\circ}$ Congresso Nacional de Cirurgia Cardiaca. Săo Paulo, SP, 2 e 3 de abril, 1993.

- Do Hospital das Clínicas da Faculdade de Medicina da Universidade de São Paulo.

* Do Instituto do Coração do Hospital das Clínicas da Faculdade de Medicina da Universidade de São Paulo.

Endereço para separatas: Ricardo Beyruti. Caixa Postal 21.480 04698-970 São Paulo, SP, Brasil.
} 
BEYRUTI, R.; STOLF, N. A. G.; MOREIRA, L. E.P.; PÊGO-FERNANDES, P. M.; MADY, C.; JATENE, A. D. - A insuficiência da valva aórtica na dissecção crônica da aorta proximal: troca ou reconstrução valvar? Rev. Bras. Cir. Cardiovasc., 8 (2):108-117, 1993.

das de forma estratificada, de acordo com seu tipo, sua localização e sua duração $2,7,12,13,18$, $21,27,28,30,31$.

Desta forma, no Instituto do Coração do Hospital das Clínicas da Faculdade de Medicina da Universidade de São Paulo, as doenças aórticas para efeito de conduta e análise de resultados têm sido divididas em dois grandes grupos, com subdivisões correspondentes: Aneurismas Verdadeiros e Dissecçōes Aórticas. Nestas, entre outros fatores utilizados para sua classificação, são considerados a presença do comprometimento da aorta ascendente (tipos I e II de DEBAKEY) e o tempo decorrido desde o aparecimento da delaminação.

Ao contrario das demais formas da doença aórtica, nos pacientes portadores de dissecções crônicas com envolvimento da aorta ascendente, a manifestação clínica predominante é a da insuficiência cardíaca congestiva decorrente da insuficiência da valva aórtica $11,12,18$. O tratamento cirúrgico destes pacientes deve, portanto, compreender, além da obliteração da falsa luz, a correção da insuficiência aórtica.

Tendo em vista que os procedimentos cirúgicos para correção da insuficiência valvar presente neste tipo de dissecção ainda não foram devidamente avaliados isoladamente, é objetivo deste trabalho analisar os resultados clínicos e ecocardiográficos tardios obtidos na correção da insuficiência valvar utilizando-se duas técnicas distintas, a substituição da valva ou sua preservação, obtida pela pexia comissural.

\section{CASUÍSTICA E MÉTODOS}

No período compreendido entre janeiro de 1980 e dezembro de 1989 , foram operados, consecutivamente, no Instituto do Coração do Hospital das Clínicas da Faculdade de Medicina da Universidade de São Paulo, 48 pacientes portadores de dissecção crônica com envolvimento da aorta ascendente, com insuficiência aórtica associada. A dissecção foi considerada crônica quando, comprovadamente, haviam transcorrido no mínimo duas semanas do episódio inicial ${ }^{12}$.

Estes pacientes foram, para efeito de análise comparativa, divididos em dois grupos de acordo com o procedimento realizado para correção da disfunção valvar: Grupo A, constituído por 24 pacientes nos quais a valva foi preservada e Grupo B, pelos 24 pacientes nos quais a valva foi substituída.

A idade dos pacientes por ocasião de suas internaçōes variava entre 23 a 73 anos, $68,7 \%$ deles com idade entre 30 e 49 anos. Trinta e nove $(81,2 \%)$ pacientes eram do sexo masculino e 9 do sexo feminino, sendo $42(87,5 \%)$ da raça branca e 6 da raça negra.

Trinta e três $(68,7 \%)$ pacientes eram portadores de dissecção tipo I da classificação de DEBAKEY e $15(31,3 \%)$ pacientes portadores de dissecção do tipo II.

Seis $(12,5 \%)$ pacientes foram operados entre 15 e 30 dias após o episódio inicial da dissecção, caracterizado basicamente pela sintomatologia dolorosa; $22(45,8 \%)$ entre o primeiro e quarto mês e $11(22,9 \%)$ pacientes após o quarto mês. No primeiro grupo encontravam-se os pacientes com maior repercussão clínica e hemodinâmica da doença. Em $9(18,7 \%)$ pacientes não foi possível caracterizar, pela história clínica ou pela ocorrência da dor, o momento do incício da dissecção aórtica. Nestes, a sintomatologia foi decorrente da insuficiência aórtica.

A etiologia predominante da dissecção aórtica foi a hipertensão arterial sistêmica, presente em 30 $(62,5 \%)$ pacientes; outros fatores etiológicos foram: a síndrome de Marfan em 10 pacientes; o trauma cirúrgico ou conseqüente a cateterismo arterial em 4; a sífilis em 1; não sendo determinada a etiologia em 3 pacientes.

Apenas $3(6,25 \%)$ pacientes encontravam-se normotensos e sem alterações hemodinâmicas quando da indicação da operação. Nos demais, 21 $(43,75 \%)$ pacientes apresentavam hipertensão arterial sistêmica; $10(20,8 \%)$ pacientes exclusivamente insuficiência cardiaca esquerda e em $14(29,1 \%)$ esta associava-se à hipertensão arterial.

Em $5(10,4 \%)$ pacientes ocorreram manifestações clínicas da dissecção. A insuficiência renal aguda, adequadamente compensada por ocasião da operação, ocorreu em 2 pacientes; 1 paciente apresentou paresia de membro superior direito; outro isquemia de membros inferiores. Um paciente apresentou dor abdominal atribuída a insuficiência arterial mesentérica. Seis pacientes apresentavam diminuição dos pulsos dos membros superiores. Em 3 deles, havia também alterações dos pulsos de membros inferiores e carotídeos. Apenas 1 paciente manifestou sinais pré-operatórios de expansão aneurismática da dissecção, ao nível da aorta abdominal. Em $4(8,3 \%)$ pacientes, havia sinais sugestivos de rotura da dissecção; em 3 , esta complicação comprometia o pericárdio; no caso remanescente, manifestou-se por hemoptise.

O grau da insuficiência aórtica foi classificado auscultatoriamente em discreto em 12 (25\%) pacientes; moderado em $23(47,9 \%)$ e severo em 13 (27\%) pacientes. 
BEYRUTI, R.; STOLF, N. A. G.; MOREIRA, L. E.P.; PÊGO-FERNANDES, P. M.; MADY, C.; JATENE, A. D. - A insuficiência da valva aórtica na dissecção crônica da aorta proximal: troca ou reconstrução valvar? Rev. Bras. Cir. Cardiovasc., 8 (2):108-117, 1993.

Todos os pacientes deste estudo apresentavam-se sintomáticos quando da indicação cirúrgica; $5(10,4 \%)$ em classe funcional grau II; $27(56,2 \%)$ em classe funcional grau III e $16(33,3 \%)$ em classe funcional grau IV, segundo a classificação da "New York Heart Association".

A operação foi realizada por mediosternotomia longitudinal, utilizando-se circulação extracorpórea convencional com canulação da artéria femoral, hipotermia moderada e proteção miocárdica com solução cardioplégica cristalóide e hipotermia tópica. Realiza a aortotomia longitudinal, o interior do vaso era examinado, procurando-se identificar o orifício de entrada da dissecção e a anatomia da valva aórtica. Não havendo grande dilatação anular, apresentando as válvulas semilunares morfologia normal e a insuficiência aórtica sendo decorrente do desabamento comissural, optava-se por sua preservação suspendendo e fixando as suas comissuras com ponto em $U$ de fio monofilamentar de Polipropileno 4-0 apoiado em almofadas de Teflon, passado no ângulo comissural, compreendendo todas as camadas dissecadas do vaso e amarrado externamente.

A aorta era seccionada circunferencialmente acima do plano da valva e distalmente próxima ao tronco braquiocefálico, as camadas dissecadas reaproximadas obliterando a dissecção através de pontos em forma de $U$ com fio monofilamentar de
Polipropileno 4-0, apoiados em tiras de feltro de Teflon colocadas uma sobre a túnica externa e aoutra dentro da luz verdadeira do vaso. A partir de 1986, passou-se a utilizar adicionalmente adesivo biológico "GRF" entre as camadas delaminadas. A valva era, então, reexaminada para assegurar a adequada coaptação das válvulas e, a seguir, interpunhase o enxerto previamente selecionado e pré-coagulado à tempeartura ambiente com sangue autógeno, anastomosado com sutura contínua de fio monofilamentar de Polipropileno 3-0.

No caso de grande dilatação do anel aórtico ou a valva com lesão primária que a comprometia estruturalmente, realizava-se sua substituição por prótese valvular associada à substituição da aorta por tubo de Dacron em posição supracoronariana ou por tubo valvulado de Dacron, no qual se implantavam os óstios coronários, como originalmente descrito por BENTALL \& DE BONO, em $1968{ }^{6}$.

Em um paciente do Grupo A e outro do Grupo B operados no inicío da série, a correção da delaminação se fez mediante obliteração do orifício de entrada sem interposição de enxerto vascular.

Os pacientes foram submetidos a avaliação ecocardiográfica pré-operatória e pós-operatória tardia, para quantificar-se o grau da insuficiência aórtica, o diâmetro aórtico e a função ventricular esquerda.

\section{COMPARAÇĀO ECOCARDIOGRÁFICA PRÉ-OPERATÓRIA DOS PARÂMETROS DA FUNÇÃO VENTRICULAR ESQUERDA} (TABELA 1) E DO GRAU DE INSUFICIÊNCIA AÓRTICA (TABELA 2) ENTRE OS GRUPOS A E B

TABELA 1

DIÂMETRO DA AORTA E PARÂMETROS DO VENTRICCULO ESQUERDO

\begin{tabular}{|c|c|c|c|c|c|c|c|c|c|}
\hline \multirow{2}{*}{\multicolumn{2}{|c|}{$\begin{array}{l}\text { Diâmetro da aorta } \\
\qquad(\mathrm{mm})\end{array}$}} & \multicolumn{8}{|c|}{ Ventrículo esquerdo } \\
\hline & & \multicolumn{2}{|c|}{$\begin{array}{l}\text { Diâmetro sistólico } \\
\qquad(\mathrm{mm})\end{array}$} & \multicolumn{2}{|c|}{$\begin{array}{l}\text { Diâmetro Diastólico } \\
\qquad(\mathrm{mm})\end{array}$} & \multicolumn{2}{|c|}{$\begin{array}{c}\text { Fração de } \\
\text { encurtamento }\end{array}$} & \multicolumn{2}{|c|}{ Fração de ejeção } \\
\hline Grupo A & Grupo B & Grupo A & Grupo B & Grupo A & Grupo B & Grupo A & Grupo B & Grupo A & Grupo B \\
\hline 41,93 & 51,47 & 38,07 & 46,47 & 59,27 & 67,65 & 0,36 & 0,32 & 0,73 & 0,68 \\
\hline$( \pm 7,85)$ & $( \pm 12,20)$ & $( \pm 7,73)$ & $( \pm 9,76)$ & $( \pm 10,04)$ & $( \pm 9,54)$ & $( \pm 0,07)$ & $( \pm 0,07)$ & $( \pm 0,06)$ & $( \pm 0,09)$ \\
\hline \multicolumn{2}{|c|}{$p=0,015$} & \multicolumn{2}{|c|}{$p=0,012$} & \multicolumn{2}{|c|}{$p=0,022$} & \multicolumn{2}{|c|}{ (NS) } & \multicolumn{2}{|c|}{ (NS) } \\
\hline
\end{tabular}

TABELA 2

INSUFICIÊNCIAAÓRTICA

\begin{tabular}{cccccccccc}
\hline \multicolumn{2}{c}{ Ausente } & \multicolumn{2}{c}{ Grau I } & \multicolumn{2}{c}{ Grau II } & \multicolumn{2}{c}{ Grau III } & \multicolumn{2}{c}{ Grau IV } \\
\hline Grupo A & Grupo B & Grupo A & Grupo B & Grupo A & Grupo B & Grupo A & Grupo B & Grupo A & Grupo B \\
\hline 0 & 1 & 6 & 0 & 3 & 3 & 6 & 9 & 0 & 4 \\
& $(5,88 \%)$ & $(40 \%)$ & & $(20 \%)$ & $(17,65 \%)$ & $(40 \%)$ & $(52,94 \%)$ & $(23,53 \%)$ \\
\hline
\end{tabular}


BEYRUTI, R.; STOLF, N. A. G.; MOREIRA, L. E.P.; PÊGO-FERNANDES, P. M.; MADY, C.; JATENE, A. D. - A insuficiência da valva aórtica na dissecção crônica da aorta proximal: troca ou reconstrução valvar? Rev. Bras. Cir. Cardiovasc., 8 (2):108-117, 1993.

A sobrevida foi calculada pelo método atuarial e as curvas comparadas pelo método do Log-Rank. As variáveis singelas foram analisadas pelo teste de Qui-quadrado e, para análise das variáveis numéricas, empregou-se o teste $T$ de Student para amostras pareadas e não pareadas, conforme indicado (KRAMER ${ }^{22}$ ).

A análise estatística mostrou serem os grupos comparáveis na distribuição de sexo, raça, classificação segundo DEBAKEY, etiologia, condição hemodinâmica pré-operatória, magnitude ausculatória da insuficiência aórtica, classe funcional e tempo de seguimento, sendo significativamente menor a média de idade dos pacientes do Grupo B (Grupo A $47,9 \pm 10,8$; Grupo B $40,2 \pm 9,5 \mathrm{p}=0,03)$. Na avaliação ecocardiográfica, os pacientes deste grupo apresentavam grau de insuficiência aórtica e diâmetros aórtico (DA) e ventriculares esquerdo sistólico e diastólico (DSVE e DDVE), significativamente maiores do que os pacientes do Grupo A, $\mathrm{p}<0,03$ (Tabelas 1 e 2 ).

\section{RESULTADOS}

\section{Mortalidade e Sobrevida}

Ocorreram $11(22,9 \%)$ óbitos entre os 48 pacientes, sendo $4(8,3 \%)$ óbitos imediatos e $7(14,5 \%)$ tardios.

No Grupo A ocorreram $5(20,83 \%)$ óbitos, sendo $3(12,5 \%)$ óbitos imediatos e $2(8,33 \%)$ tardios. Dos óbitos imediatos, 2 foram decorrentes da síndrome do baixo débito cardíaco pós-operatório e 1 conseqüênte a complicações pulmonares e a insuficiência respiratória que se seguiram a distúrbios da coagulação e politransfusão sangüínea. As causas que levaram aos óbitos tardios foram a insuficiência cardíaca congestiva progressiva em 1 paciente, ocorrida aos 18 meses de seguimento, não atribuível à disfunção valvar, e o infarto agudo do miocárdio no outro, aos 59 meses de seguimento. Três pacientes foram perdidos em seu seguimento clínico, e a sobrevida cumulativa aos 7 anos, respectivamente, nesse grupo, foi de $75,75 \% \pm 8,12 \%$.

No Grupo B ocorreram 6 (25\%) óbitos, sendo $1(4,17 \%)$ óbito imediato e $5(20,83 \%)$ tardios. O óbito imediato foi decorrente de sangramento intraoperatório incontrolável. As causas que levaram aos 5 óbitos tardios foram a insuficiência cardíaca congestiva progressiva em 2 pacientes, ocorrido aos 12 e aos 90 meses de seguimento, o infarto agudo do miocárdio em 1 paciente, aos 113 meses de seguimento, e a morte súbita em outros dois, aos 6 e 14 meses de seguimento, respectivamen- te, não sendo possível determinar sua causa. A sobrevida cumulativa aos 7 anos, nesse grupo, foi de $82,72 \% \pm 7,87 \%$, não havendo diferença estatística na sobrevida atuarial entre os 2 grupos (Gráfico 1).

Os 2 pacientes nos quais a aorta ascendente não foi substituída apresentaram complicações tardias, ocorrendo expansão aneurismática aos 45 meses de seguimento em 1 e redissecção aos 60 meses no outro, sendo ambos submetidos a reoperação.

\section{Avaliação Clínica}

Nos dois grupos houve regressão pós-operatória significativa da dispnéia, persistindo sintomatologia aos pequenos esforços em apenas 1 paciente do Grupo A, portador de doença pulmonar obstrutiva crônica. Comportamento semelhante foi observado em relação à classe funcional (NYHA). Um paciente do Grupo A permaneceu em classe funcional grau III no pós-operatório tardio; os demais encontravam-se, por ocasião da avaliação, nas classes I e II. Nos pacientes dos dois grupos houve regressão pós-operatória significativa da dor torácica e da insuficiência aórtica avaliada pela ausculta e normalização ou queda nos níveis da hipertensão arterial sistêmica. A comparação destes parâmetros clínicos mostrou resposta pós-operatória semelhante nos dois grupos, não havendo significância estatística pelo teste de Qui-quadrado.

\section{Avaliação Ecocardiográfica}

Nos pacientes do Grupo A ocorreu redução pós-operatória significativa somente no DDVE $(59,27$

\section{GRÁFICO 1}

SOBREVIDA ATUARIAL DOS DOIS GRUPOS, NÃO HAVENDO DIFERENCAA ESTATIISTICA ENTRE ELES

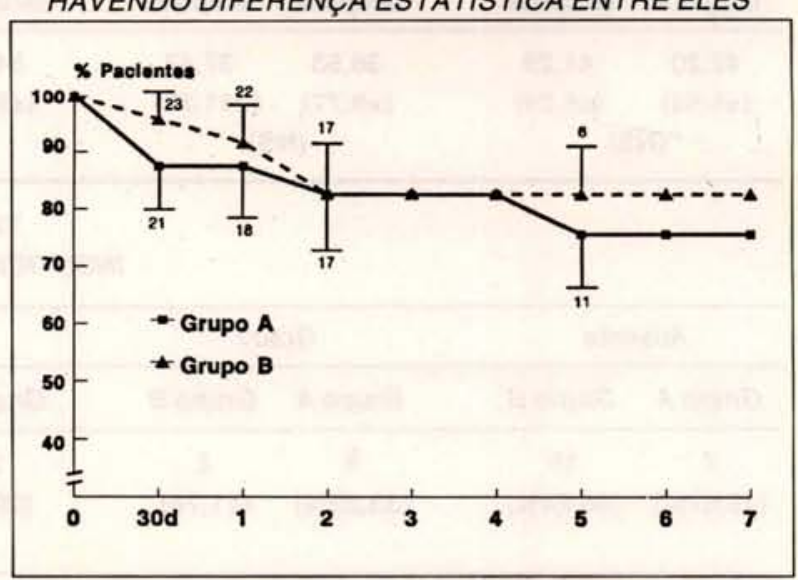


BEYRUTI, R.; STOLF, N. A. G.; MOREIRA, L. E.P.; PÊGO-FERNANDES, P. M.; MADY, C.; JATENE, A. D. - A insuficiência da valva aórtica na dissecção crônica da aorta proximal: troca ou reconstrução valvar? Rev. Bras. Cir. Cardiovasc., 8 (2):108-117, 1993.

$\mathrm{mm} \pm 10,04 \mathrm{~mm}$ verso $54,73 \mathrm{~mm} \pm 9,78 \mathrm{~mm}$, $\mathrm{p}=0,03$ ). Já nos pacientes do Grupo B, após a operação, houve diminuição do DA $(51,47 \mathrm{~mm} \pm$ $12,20 \mathrm{~mm}$ verso $41,29 \mathrm{~mm} \pm 6,09, \mathrm{p}=0,01)$, do DSVE $(46,47 \mathrm{~mm} \pm 9,76 \mathrm{~mm}$ verso $37,47 \mathrm{~mm} \pm$ $11,37 \mathrm{~mm}, \mathrm{p}=0,01)$ e do DDVE $(67,65 \mathrm{~mm}$ de $\pm 9,54$ $\mathrm{mm}$ verso $53,94 \mathrm{~mm} \pm 9,07 \mathrm{~mm}, \mathrm{p}<0,001)$. A comparação estatística dos valores pós-operatórios acima pelo teste $T$ de Student para amostras não pareadas não evidenciou diferença significativa entre os dois grupos em nenhum destes parâmetros ( $\mathrm{Ta}$ bela 3 ).

Nos dois grupos houve significativa redução pós-operatória da magnitude da insuficiência aórtica analisada ecocardiograficamente. Sete $(46,67 \%)$ pacientes do Grupo A e $15(88,24 \%)$ pacientes do Grupo B não manifestaram insuficiência aórtica, após a operação, à ecocardiografia e os demais pacientes deste grupo apresentaram a disfunção classificada em grau I $2(11,7 \%)$ pacientes. Cinco $(33,33 \%)$ pacientes do Grupo A apresentaram disfunção grau I e outros $3(20 \%)$ pacientes permaneceram com a insuficiência valvar classificada pela ecocardiografia em grau II. A comparação estatística destes parâmetros pelo teste de Qui-quidrado evidenciou que a insuficiência aórtica pós-operatória quantificada pela ecocardiografia foi significativamente maior no Grupo A do que no Grupo B $(p=0,029)$ (Tabela 4).

\section{COMENTÁRIOS}

Os principais objetivos do tratamento operatório das dissecções da aorta com comprometimento de sua porção ascendente são, na sua fase aguda, prevenir a morte do paciente determinada por eventual rotura intrapericárdica, restabelecer o fluxo sangüíneo em artérias que se ocluíram e corrigir a disfunção da valva aórtica coexistente (KIRKLIN \& BARRAT-BOYES ${ }^{19}$ ). Estes mesmos princípios norteiam o tratamento na fase crônica.

Vários fatores prognósticos importantes das dissecções aórticas, dentre eles a alta mortalidade que se seguia ao comprometimento da aorta ascendente, embora há muito conhecidos, eram desconsiderados na elaboração das publicações $16,29,33$. Tanto nas séries de pacientes submetidos a cirurgia, como nas séries de pacientes submetidos a tratamento clínico, avaliavam-se globalmente pacientes portadores de aneurismas verdadeiros, de dissecções da aorta ascendente e da aorta descendente; pacientes portadores de dissecções agudas e crônicas, ou então pacientes com os mais variados tipos e graus de complicações $5,8,17,39,40$. Estes aspectos impossibilitavam uma adequada análise e real avaliação dos resultados obtidos com os tratamentos propostos.

Do total de pacientes operados para correção de afecções da aorta no Instituto do Coração do

COMPARAÇĀO ECOCARDIOGRÁFICA PÓS-OPERATÓRIA DOS PARÂMETROS DA FUNÇĀO VENTRICULAR ESQUERDA (TABELA 3) E DO GRAU DE INSUFICIÊNCIA AÓRTICA (TABELA 4) ENTRE OS GRUPOS A E B

TABELA 3

DIÂMETRO DA AORTA E PARÂMETROS DO VENTRÍCULO ESQUERDO

\begin{tabular}{|c|c|c|c|c|c|c|c|c|c|}
\hline \multirow{2}{*}{\multicolumn{2}{|c|}{$\begin{array}{l}\text { Diâmetro da aorta } \\
\qquad(\mathrm{mm})\end{array}$}} & \multicolumn{8}{|c|}{ Ventrículo esquerdo } \\
\hline & & \multicolumn{2}{|c|}{$\begin{array}{c}\text { Diâmetro sistólico } \\
(\mathrm{mm})\end{array}$} & \multicolumn{2}{|c|}{$\begin{array}{l}\text { Diâmetro Diastólico } \\
\qquad(\mathrm{mm})\end{array}$} & \multicolumn{2}{|c|}{$\begin{array}{c}\text { Fração de } \\
\text { encurtamento }\end{array}$} & \multicolumn{2}{|c|}{ Fraçāo de ejeçāo } \\
\hline Grupo A & Grupo B & Grupo A & Grupo B & Grupo A & Grupo B & Grupo A & Grupo B & Grupo A & Grupo B \\
\hline $\begin{array}{c}42,20 \\
( \pm 5,64)\end{array}$ & $\begin{array}{c}41,29 \\
( \pm 6,09)\end{array}$ & $\begin{array}{c}36,53 \\
( \pm 9,77)\end{array}$ & $\begin{array}{r}37,47 \\
( \pm 11,37)\end{array}$ & $\begin{array}{c}54,73 \\
( \pm 9,78)\end{array}$ & $\begin{array}{c}53,94 \\
( \pm 9,07)\end{array}$ & $\begin{array}{c}0,34 \\
( \pm 0,07)\end{array}$ & $\begin{array}{c}0,32 \\
( \pm 0,09)\end{array}$ & $\begin{array}{c}0,70 \\
( \pm 0,06)\end{array}$ & $\begin{array}{c}0,67 \\
( \pm 0,14)\end{array}$ \\
\hline \multicolumn{2}{|c|}{ (NS) } & \multicolumn{2}{|c|}{ (NS) } & \multicolumn{2}{|c|}{ (NS) } & \multicolumn{2}{|c|}{ (NS) } & \multicolumn{2}{|c|}{ (NS) } \\
\hline & & & & $\begin{array}{l}\text { TABEL } \\
\text { FICIÊNCI }\end{array}$ & 4 & & & & \\
\hline \multicolumn{2}{|c|}{ Ausente } & \multicolumn{2}{|c|}{ Grau I } & \multicolumn{2}{|c|}{ Grau II } & \multicolumn{2}{|c|}{ Grau III } & \multicolumn{2}{|c|}{ Grau IV } \\
\hline Grupo A & Grupo B & Grupo A & Grupo B & Grupo A & Grupo B & Grupo A & Grupo B & Grupo A & Grupo B \\
\hline $\begin{array}{c}7 \\
(46,67 \%)\end{array}$ & $\begin{array}{c}15 \\
(88,24 \%)\end{array}$ & $\begin{array}{c}5 \\
(33,33 \%)\end{array}$ & $\begin{array}{c}2 \\
(11,76)\end{array}$ & $\begin{array}{c}3 \\
(20 \%)\end{array}$ & 0 & 0 & 0 & 0 & 0 \\
\hline$(46,67 \%)$ & & \multicolumn{8}{|c|}{$p=0,029$} \\
\hline
\end{tabular}


BEYRUTI, R.; STOLF, N. A. G.; MOREIRA, L. E. P.; PÊGO-FERNANDES, P. M.; MADY, C.; JATENE, A. D. - A insuficiência da valva aórtica na dissecção crônica da aorta proximal: troca ou reconstrução valvar? Rev. Bras. Cir. Cardiovasc., 8 (2):108-117, 1993.

Hospital das Clínicas da Faculdade de Medicina da Universidade de São Paulo, foram selecionados para este estudo somente os que apresentassem insuficiência aórtica decorrente de dissecções crônicas da aorta ascendente. Este critério baseou-se nas particularidades clínicas e evolutivas desses pacientes. $O$ prognóstico daqueles em que a aorta ascendente encontra-se envolvida é muito mais grave quando comparado àqueles em que este segmento mantem-se íntegro $2,11,27$. Por outro lado, na fase crônica da doença, os pacientes são operados em condições de estabilidade hemodinâmica e com controle adequado de eventuais complicações orgânicas, o que determina menor morbimortalidade operatória, melhor prognóstico e maior sobrevida tardia em comparação aos pacientes operados na face aguda $2,13,11,27$. A insuficiência cardíaca congestiva predomina na fase crônica, sendo esta decorrente da insuficiência aórtica 12 . DOROGHAZI et alii ${ }^{13}$, ao analisarem a mortalidade hospitalar e a sobrevida tardia de 160 pacientes portadores de dissecção aórtica, concluíram que a apresentação crônica foi o fator determinante mais importante da sobrevida imediata e tardia e que, na dissecção aguda, o prognóstico foi determinado fundamentalmente pelas complicações associadas, das quais somente a insuficiência aórtica não exercia influência desfavorável na evolução dos pacientes. Para KOUCHOUKOS et alii ${ }^{21}$, a necessidade de operação imediata na dissecção aguda foi a única variável independente relacionada com a maior mortalidade hospitalar. JEX et alii ${ }^{18}$, além de confirmarem a maior prevalência da insuficiência aórtica e da insuficiência cardiaca congestiva nos pacientes crônicos, relataram mortalidade imediata em $37 \%$ dos pacientes operados na fase aguda e somente em $9 \%$ dos operados na fase crônica. Resultados semelhantes foram descritos por MOREIRA et alii 28, que, analisando casuística desta Instituição, observaram expressiva redução na mortalidade hospitalar nos pacientes operados na fase crônica em relação aos operados na fase aguda, e por RAUDKIVI et alii ${ }^{31}$. Apenas para HAVERICH et alii ${ }^{15}$, o risco operatório e a sobrevida tardia independeram da precocidade da operação e do tipo da dissecção.

A melhor forma de se tratar a insuficiência aórtica na dissecção da aorta é controversa. Sua fisiopatologia tem particularidades bastante diferentes das disfunções causadas por outras etiologias. Ela decorre da delaminação das camadas da parede da aorta e da extensão do hematoma até o anel valvar aórtico, determinando perda de sustentação das válvulas semilunares e de suas comissuras. Os elementos anatômicos do aparelho valvar aórtico geralmente não exibem as alterações histopatológicas características das lesões de origem reumática ou aterosclerótica $16,23,24$. Desta forma, a morfologia e a integridade da valva aórtica podem ser restabelecidas cirurgicamente. Mediante técnicas reconstrutoras, como a utilizada neste estudo, é possível, em determinadas situações, preservar a valva natural, evitando-se, assim, todos os inconvenientes associados à troca valvar por substitutos mecânicos ou biológicos. Se na dissecção aguda da aorta tem sido conduta comum a preservação da valva aórtica, na dissecção crônica a maior parte das publicações mostra que a freqüência da substituição da valva é muito alta $9,11,15,18,20,27,28,35$. Para DOROGHAZI et alii ${ }^{13}$, a menor aplicação da plastia na fase crônica deve-se a alterações histopatológicas rapidamente progressivas, que ocrrem no aparelho valvar submetido a condições de funcionamento anormais o que o tornam inadequado para a reconstrução. Vários autores procuram destacar que, quando possível, a valva aórtica deve ser preservada sem, no entanto, caracterizarem em suas publicações os motivos que os levaram a optar pela plastia valvar $1,2,10,11,15,34,35,37$. Para KOSTER et alii ${ }^{20}$, a valva aórtica deve, sempre, ser preservada na dissecção aguda. MENG et alii ${ }^{26}$ concluem que, quando o funcionamento normal da valva aórtica pode ser restabelecido, a preservação da valva natural é preferivel à substituição. Por outro lado, vários autores sugerem que a manutenção de tecido aórtico justa e/ou supra-anular pode ser sede de futuras dilatações ou redissecções, principalmente na síndrome de Marfan, e recomendam o emprego de técnicas mais radicais que incluem, além da troca valvar, a substituição de toda a aorta ascendente ${ }^{14}$. 21,25 .

São escassas as publicações referentes à preservação da valva aórtica na dissecção crônica 20 , $26,30,38$. JEX et alii ${ }^{18}$, analisando um grupo de 121 pacientes operados consecutivamente devido a dissecções agudas e crônicas da aorta, compararam os resultados da suspensão valvar realizada em 18 deles na fase crônica, com os resultados da substituição valvar em 58 pacientes semelhantes, não tendo observado diferenças quanto à mortalidade imediata, ou complicações e sobrevida tardia. Pelos bons resultados obtidos com a preservação da valva aórtica, estes autores sugerem que esta técnica seja a de escolha para a correção da insuficiência aórtica.

Com a crescente experiência e confiança adquiridas com a valvoplastia nas dissecçōes agudas, passamos a adotar esta técnica nos casos crônicos, observando alguns critérios para sua aplicação. Desta forma, na presença de grandes dilatações ânulo-aórticas, de comprometimento valvar primário e, mesmo, na síndrome de Marfan, optamos por procedimento que inclua a substituição valvar. Esta abordagem pode explicar as diferenças pré-operatórias entre os dois grupos desta casuística rela- 
BEYRUTI, R.; STOLF, N. A. G.; MOREIRA, L. E.P.; PÊGO-FERNANDES, P. M.; MADY, C.; JATENE, A. D. - A insuficiência da valva aórtica na dissecção crônica da aorta proximal: troca ou reconstrução valvar? Rev. Bras. Cir. Cardiovasc., 8 (2):108-117, 1993.

cionadas ao maior diâmetro da aorta e à severidade da insuficiência aórtica e sua repercussão sobre o ventrículo esquerdo, observadas nos pacientes do Grupo B.

Os resultados observados na evolução pósoperatória evidenciaram que a sobrevida e a melhora clínica foram semelhantes com as duas técnicas, não ocorrendo qualquer tipo de repercussão imediata ou tardia atribuível a insuficiência aórtica residual detectada à ecocardiografia nos pacientes do Grupo A, a exemplo do que relatam AUSTEN et alii ${ }^{3}$ e KIRKLIN \& BARRAT-BOYES ${ }^{19}$. No seguimento tardio não houve sua progressão ou recidiva, indicando que a funcionalidade valvar obtida pela plastia se mantém a longo prazo. Tão pouco foram observadas complicações tromboembólicas ou hemorrágicas nos pacientes do Grupo B. Não ocorreram dilatações ou redissecções no tecido aórtico supracoronariano remanescente nos pacientes nem a aorta ascendente foi substituída. Estas complicações ocorreram somente nos 2 pacientes em que se realizou a aortoplastia, não devendo, portanto, esta técnica ser empregada nesta afecção 25,36 . O emprego do adesivo biológico "GRF" utilizado na segunda metade da experiência pode, segundo BACHET et alii ${ }^{4}$, contribuir favoravelmente no sentido de evitar este tipo de ocorrência, por conferir maior resistência à parede aórtica tratada.

Em conclusão, acreditamos que:

- A dissecção crônica da aorta com insuficiência aórtica associada pode ser tratada com baixa mortalidade hospitalar e tardia, determinando nítida melhora clínica e funcional dos doentes e estes resultados são igualmente obtidos com as duas técnicas estudadas.

- Existem, neste grupo de pacientes, diferenças anatomoclínicas e fisiopatológicas que devem ser identificadas para a adequada escolha da técnica de correção da disfunção valvar.

- A boa evolução clínica e a ausência de complicações a longo prazo, dos pacientes nos quais se realizou a preservação valvar, indicam que este procedimento deva constituir a primeira opção do cirurgião na correção da insuficiência aórtica associada à dissecção crônica da aorta.

BEYRUTI, R.; STOLF, N. A. G.; MOREIRA, L. F. P.; PÊGO-FERNANDES, P. M.; MADY, C.; JATENE, A. $D$. - Aortic valve insufficiency in chronic aortic dissection: valve replacement or reconstruction? Rev. Bras. Cir. Cardiovasc., 8(2): 108-117, 1993.

ABSTRACT: From January 1980 to December 1989, 48 consecutive patients with chronic aortic dissection and aortic valve insufficiency underwent operation. They were analyzed in 2 groups (24 patients each) to evaluate the late clinical and echocardiographic outcome of those in whom the aortic valve was preserved (Group A) compared with those having valve replacement (Group B). The overall preoperative characteristics of the two groups were similar except for the age (Group A 47.9 \pm 10.8 versus Group B 40.2 \pm 9.5 years, $p=0.03$ ). In the preoperative echocardiographic evaluation, Group $B$ patients had significantly higher aortic root and left ventricle systolic and diastolic diameters $(p<0.03)$, and aortic insufficiency grade $(p=0.02)$. The hospital mortality was $12.5 \%$ in Group $A$ and $4.2 \%$ in Group B and the seven years actuarial survival rate was respectively $75.7 \% \pm 9.8 \%$ and $82.7 \% \pm 7.9 \%$. Postoperatively there was, in both groups, a significant improvement in all clinical parameters evaluated (dyspnea, chest pain, arterial hypertension, aortic insufficiency murmurs $(p<0.001)$. There was also a marked reduction in the aortic root and left ventricle diameters in all patients compared to preoperative values $(p<0.05)$ without statistical difference between the groups. Echocardiographic mild aortic insufficiency remained in $20 \%$ of the patients in whom the valve was preserved, without long term hemodynamic or clinical repercussion. Hence, we conclude that aortic insufficiency in chronic dissection can be equally treated with both techniques. Aortic valve preservation should, whenever possible, be attempted and considered as the procedure of choice.

DESCRIPTORS: heart valves, aortic, surgery; heart valves, surgery. 
BEYRUTI, R.; STOLF, N. A. G.; MOREIRA, L. E. P.; PÊGO-FERNANDES, P. M.; MADY, C.; JATENE, A. D. - A insuficiência da valva aórtica na dissecção crônica da aorta proximal: troca ou reconstrução valvar? Rev. Bras. Cir. Cardiovasc., 8 (2):108-117, 1993.

\section{REFERÊNCIAS BIBLIOGRÁFICAS}

1 ANAGNOSTOPOULOS, C. E.; PRABHAKAR, M. J. S.; KITTLE, C. F. - Aortic dissections and dissecting aneurysms. Am. J. Cardiol., 30: 263-273, 1972.

2 APPELBAUM, A.; KARP, R. B.; KIRKLIN, J. W. - Ascending vs. descending aortic dissections. Ann. Surg., 183: 296-300, 1976.

3 AUSTEN, W. G.; BUCKLEY, M. J.; MCFARLAND, J.; DESANCTIS, R. W.; SANDERS, C. A. - Therapy of dissecting aneurysms. Arch. Surg., 95: 835-842, 1967.

4 BACHET, J.; GIGOU, F.; LAURIAN, C.; BICAL, O.; GOUDOT, B.; GUILMET, D. - Four-year clinical experience with the Gelatin - Resorcine-Formol biological glue in acute aortic dissection. J. Thorac. Cardiovasc. Surg., 83: 212-217, 1982.

5 BECKWITH, J. R.; MULLER, W. H.; WARREN, D.; WOOD, J. E. - Acute dissecting aneurysm of the aorta. Arch. Intern. Med., 104: 217-225, 1959.

6 BENTALL, H. \& DE BONO, A. - A technique for complete replacement ofthe ascending aorta. Thorax, 33: 338-339, 1968.

7 DAILY, P. O.; TRUEBLOOD, H. W.; STINSON, E. B.; WUERFLEIN, R. D.; SHUMWAY, N. E. - Management of acute aortic dissections. Ann. Thorac. Surg., 10: 237-247, 1970

8 DEBAKEY, M. E.; COOLEY, D. A.; CREECH Jr., O. Surgical considerations of dissecting aneurysm of the aorta. Ann. Surg., 142: 586-612, 1955.

9 DEBAKEY, M. E.; HENLY, W. S.; COOLEY, D. A.; MORRIS Jr., G. C.; CRAWFORD, E. S.; BEALL Jr., A. C. - Surgical management of dissecting aneurysm involving the ascending aorta. J. Cardiovasc. Surg., 5: 200-211, 1964.

10 DEBAKEY, M. E.; HENLY, W. S.; COOLEY, D. A.; MORRIS Jr., G. C.; CRAWFORD, E. S.; BEALL Jr., A. C. - Surgical management of dissecting aneurysms of the aorta. J. Thorac. Cardiovasc. Surg., 49: 130$149,1965$.

11 DEBAKEY, M. E.; MCCOLLUM, C. H.; CRAWFORD, E. S.; MORRIS Jr., G. C.; HOWELL, J.; NOON, G. P.; LAWRIE, G. - Dissection and dissecting aneurysms of the aorta: twenty-year follow-up of five hundred twenty-seven patients treated surgically. Surgery, 92: 1118-1143, 1982.

12 DOROGHAZI, R. M. \& SLATER, E. E. - Aortic dissection. New York, McGraw-Hill Book Company, 1983.

13 DOROGHAZI, R. M.; SLATER, E. E.; DESANCTIS, R. W.; BUCKLEY, M. J. - Long-term survival of patients with treated aortic dissection. J. Am. Coll. Cardiol., 3: 1026-1034, 1984.

14 GREY, D. P.; OTT, D. A.; COOLEY, D. A. - Surgical treatment of aneurysm of the ascending aorta with aortic insufficiency: a selective approach. J. Thorac. Cardiovasc. Surg., 86: 864-877, 1983.

15 HAVERICH, A.; MILLER, D. C.; SCOTT, W. C.; MITCHELL, R. S.; OYER, P. E. - Acute and chronic aortic dissections: determinants of long-term outcome for operative survivors. Circulation, 72 (Supl 2): 22-34, 1985.

16 HIRST Jr., A. E.; JOHNS Jr., J. V.; KIME Jr., S. W. Dissecting aneurysm of the aorta: a review of 505 cases. Medicine (Baltimore), 37: 217-279, 1958.

17 HUME, D. C. \& PORTER, R. R. - Acute dissecting aneurysms. Surgery, 53: 122-154, 1963.

18 JEX, R. K.; SCHAFF, H. V.; PIEHLER, J. M.; ORSZULAK, T. A.; PUGA, F. J. - Repair of ascending aortic dissection: influence of associated aortic valve insufficiency on early and late results. J. Thorac. Cardiovasc., 93: 375-384, 1987.

19 KIRKLIN, J. W. \& BARRAT-BOYES, B. G. - Cardiac surgery. New York, John Wiley and Sons, 1986. p. 1471-1525.

20 KOSTER, J. K.; COHN, L. H.; MEE, R. B. B.; COLLINS Jr., J. J. - Late results of operation for acute aortic dissection producing aortic insufficiency. Ann. Thorac. Surg., 26: 461-467, 1978.

21 KOUCHOUKOS, N. T.; MARSHALL Jr., W. G.;WEDIGESTECHER, T. A. - Eleven-year experience with composite graft replacement of the ascending aorta and aortic valve. J. Thorac. Cardiovasc. Surg., 92: 691-705, 1986.

22 KRAMER, M. S. - Clinical epidemiology and biostatistics: a primer for clinical investigators and decision makers. Berlin, Sringer-Verlag, 1988. 286 p.

LARSON, E. W. \& EDWARDS, W. D. - Risk factors for aortic dissection: a necropsy study of 161 cases. Am. J. Cardiol., 53: 849-855, 1984.

24 LEU, H. J. \& JULKE, M. - Thoracic aortic aneurysm: pathologico-anatomical analysis of 111 cases. Schweiz. Med. Wochenschr., 114: 593-595, 1984.

25 McCREADY, R. A. \& PLUTH, J. R. - Surgical treatment of ascending aortic aneurysms associated with aortic valve insufficiency. Ann. Thorac., 28: 206-216, 1979.

26 MENG, R. L.; NAJAFI, H.; JAVID, H.; HUNTER, J. A.; GOLDIN, M. D. - Acute ascending aortic dissection: surgical management. Circulation, 64 (Supl. 2): 231 $234,1981$.

27 MILLER, D. C.; STINSON, E. B.; OYER, P. E.; ROSSITER, S. J.; REITZ, B. A. - Operative treatment of aortic dissections: experience with 125 patients over a sixteen-year period. J. Thorac. cardiovasc. Surg., 78: 356-382, 1979. 
BEYRUTI, R.; STOLF, N. A. G.; MOREIRA, L. E. P.; PÊGO-FERNANDES, P. M.; MADY, C.; JATENE, A. D. - A insuficiência da valva aórtica na disseç̧ão crônica da aorta proximal: troca ou reconstrução valvar? Rev. Bras. Cir. Cardiovasc., 8 (2):108-117, 1993.

MOREIRA, L. F. P.; STOLF, N. A. G.; VIANNA, C. B. - Fatores de risco na cirurgia de dissecçōes da aorta ascendente e arco aórtico. Rev. Bras. Circardiovasc., 2: 121-128, 1987.

PEACOCK, T. B. - Cases of dissecting aneurysm, or that form of aneurysmal affection in which the sac is situated between the coats of the vessel. Edinburgh Med. Surg. J., 60: 276, 1843. ApudDOROGHAZI, R. M. \& SLATER, E. E., 1983.

PÊGO-FERNANDES, P. M.; STOLF, N. A. G.; MOREIRA, L. F. P.; BARRETO, A. C. P.; BITTENCOURT, D.; JATENE, A. D. - Management of aortic insufficiency in chronic aortic dissection. Ann. Thorac. Surg., 51: 438-442, 1991.

31 RAUDKIVI, P. J.; WILLIAMS, J. D.; MONRO, J. L.; ROSS, J. K. - Surgical treatment of the ascending aorta: fourteen years experience with 83 patients. J. Thorac. Cardiovasc. Surg., 98 (Parte 1): 675-682, 1989.

REUL Jr., G. J.; COOLEY, D. A.; HALLMAN, G. L.; REDDY, S. B.; KYGER III, E. R.; WUKASCH, D. C. - Dissecting aneurysm of the descending aorta. Arch. Surg., 110: 632-640, 1975.

SHENNAN, T. - Dissecting aneurysms. Special Report. Series $n^{\circ}$ 193. Medical Research Council, London, His Majesty's Stationery, 1934, Apud DOROGHAZI, R. M. \& SLATER, E. E., 1983.

STOLF, N. A.; PÊGO-FERNANDES, P.; COSTA, R.; BARRETO, A. C. - Tratamento cirúrgico das dissecçōes crônicas da aorta torácica. Rev. Hosp. Clin. Fac. Med. São Paulo., 38: 3-7, 1983.

35 STRONG, W. W.; MOGGIO, R. A.; STANSEL Jr., H. G. - Acute aortic dissection: twelve-year medical and surgical experience. J. Thorac. Cardiovasc. Surg., 69: 815-821, 1974.

36 SYMBAS, P. N.; RAIZNER, A. E.; TYRAS, D. H.; HATCHER Jr., C. R.; INGLESBY, T. V.; BALDWIN, B. J. - Aneurysms of all sinuses of Valsava in patients with Marfan's syndrome: an unusual late complication following replacement of aortic valve and ascending aorta for aortic regurgitation and fusiform aneurysm of ascending aorta. Ann. Surg., 174: 902-907, 1971.

37 VILJANEN, T.; LANDTMAN, M.; LUOSTO, R. - Late results of the surgical treatment for aortic dissections. Thorac. Cardiovasc. Surg., 33: 8-15, 1985.

WEBB, W. R.; ECKER, R. R.; HOLLAND, R. H.; SUGG, W. L. - Aortic aneurysm with aortic insufficiency. Am. J. Cardiol., 26: 416-418, 1970.

WHEAT Jr., M. W.; HARRIS, P. D.; MALM, J. R.; KAISER, G.; BOWMAN Jr., F. O.; PALMER, R. F. Acute dissecting aneurysms of the aorta: treatment and results in 64 patients. J. Thorac. Cardiovasc. Surg., 58: 344-351, 1969.
40 WHEAT Jr., M. W.; PALMER, R. F.; BARTLEY, T. D.; SEELMAN, R. C. - Treatment of dissecting aneurysms of the aorta without surgery. J. Thorac. Cardiovasc. Surg., 50: 364-373, 1965.

\section{Discussão}

\section{DR. ANTONINHO S. ARNONI \\ São Paulo, SP}

Eu quero parabenizar o Dr. Beyruti e o grupo do InCór pelo seu trabalho e agradecer à Comissão Organizadora a honra de comentá-lo. No Instituto Dante Pazzanese de Cardiologia, a experiência com dissecções crônicas da aorta é semelhante à do InCór, com 67 pacientes operados de 1984 a 1991. Mas, seguindo a orientação deste Congresso, não vou comentar nossos números, e apenas me ater ao trabalho do Dr. Beyruti, formulando algumas questões de dúvidas que tive ao ler seu trabalho: 1) sabemos que o emprego da ecocardiográfia transoperatória tem contribuído na realização de plastias valvares. Vocês têm usado tal procedimento para as dissecções e, em caso afirmativo, alguma vez houve necessidade de mudança da tática cirúrgica, ou seja, foi preciso trocar a valva, quando a impressão do cirurgião era de que a correção tinha ficado boa? 2) A literatura classifica como crônicos os pacientes com 15 dias ou mais do episódio agudo. Entretanto, a experiência nos tem mostrado que, nos primeiros 30 dias, as estruturas ainda são muito friáveis, bem diferentes daqueles operados com 4 meses ou mais da dissecção. $\mathrm{Na}$ sua casuística, existem 6 pacientes operados entre 15 e 30 dias. Vocês encontraram alguma diferença no que diz respeito à mortalidade ou à técnica de correção? Terá sido mais fácil, nesses casos, fazer a ressuspensão? 3) A mortalidade imediata foi $12,5 \%$ para pacientes do Grupo A e $4,17 \%$ para os do Grupo B. Dois dos óbitos do Grupo A ocorreram por baixo débito. Gostaria de saber se, nesses casos, ficou algum grau de insuficiência aórtica e se ela teria contribuído para o óbito. 4) O grupo do Instituto do Coração de São Paulo publicou, em 1991, no "Annals of Thoracic Surgery", um trabalho onde analisa a evolução de 44 pacientes operados com dissecção crônica de aorta e insuficiência aórtica, entre janeiro de 1980 e dezembro de 1988. No trabalho agora apresentado, são analisados 48 pacientes de janeiro de 1980 a dezembro de 1989 . Acredito que os 44 pacientes daquele artigo façam parte de sua casuística. Isso me fez comparar as curvas atuariais dos 2 trabalhos e, apesar de ambas não mostrarem diferenças estatísticas entre os dois grupos, o que podemos ver é que, aos 60 meses, tinhamos $90 \%$ de sobrevida para o Grupo A, contra 
BEYRUTI, R.; STOLF, N. A. G.; MOREIRA, L. E. P.; PÊGO-FERNANDES, P. M.; MADY, C.; JATENE, A. D. - A insuficiência da valva aórtica na dissecção crônica da aorta proximal: troca ou reconstrução valvar? Rev. Bras. Cir. Cardiovasc., 8 (2):108-117, 1993.

cerca de 75 a $80 \%$ para o Grupo B. Agora, com 84 meses, temos o inverso: $75 \%$ para o Grupo A, contra $82 \%$ para o Grupo B, ou seja, apesar de ainda não significativo do ponto de vista estatístico, houve uma mudança das curvas, com a sobrevida sendo maior para os pacientes com substituição valvar. Isso poderia ser explicado pela insuficiência aórtica residual, que é estatisticamente maior nos pacientes do Grupo A? Desta forma, gostaria de concluir dizendo que a conservação da valva deve uma meta a ser atingida sempre, mas que não devemos temer pela substituição quando necessária, evitando deixar insuficiências residuais que possam comprometer a evolução a longo prazo. Mais uma vez queria parabenizar o $\mathrm{Dr}$. Beyruti e seus colaboradores por levantarem um problema tão atual.

\section{DR. BEYRUTI}

(Encerrando)

Prezado Dr. Arnoni: inicialmente, quero agradecer-lhe em nome do InCór e no meu, os elogios feitos, principalmente por se tratar de cirurgião de reconhecida experiência neste tipo de afecção. Respondendo às suas perguntas: 1) fazem parte do presente trabalho pacientes operados desde 1982, quando, então, não dispúnhamos ainda para uso clínico da ecocardiografia transoperatória. No InCór, mais especificamente, esta metodologia passou a estar disponível há aproximadamente 4 ou 5 anos; porém seu maior uso se faz não neste tipo de pacientes, mas sim naqueles submetidos à correção de disfunções mitrais. Assim, não me recordo de seu emprego nesta série de doentes e, portanto, a qualidade da reconstrução valvar ficou sempre a critério do cirurgião. 2) Os critérios que utilizamos para classificar em crônicas as dissecções aórticas, como foi mencionado, são aqueles universalmente aceitos e citados na literatura. $\mathrm{Na}$ série apresentada, 3 dos 4 óbitos hospitalares ocorreram nos dois primeiros anos do estudo. Embora todos os 3 tenham sido operados com mais de 4 meses de evolução ( 1 troca e 2 resuspensões), não tenho elementos que me permitam concluir que estes pacientes apresentem mortalidade superior aos operados mais precocemente e tecnicamente não observamos diferenças significativas entre eles, o que pode ser comprovado pela distribuição homogênea dos pacientes nos dois grupos quanto ao tempo de evolução da doença. Neste tipo de análise alguns fatores devem ser considerados, pois, nos anos subseqüentes, pacientes semelhantes foram operados, com mortalidade hospitalar inferior. Entre estes fatores, podemos considerar o maior risco a que se expõem os pacientes operados na fase inicial da curva de aprendizado do procedimento e, possivelmente, as facilidades técnicas obtidas com o uso do adesivo biológico GRF a partir de 1986. 3) Nos dois óbitos ocorridos por baixo débito nos pacientes do Grupo A, não havia evidências de insuficiência aórtica significativa. 4) Sem dúvida, os 44 pacientes do trabalho publicado no "Annals of Thoracic Surgery" compoem o grupo de 48 pacientes apresentados. Trata-se, portanto, de uma atualização da casuística. Assim, além do acréscimo de 4 pacientes, o que representa aproximadamente $10 \%$ da casuística anterior, as informações atualizadas sobre seguimento imediato e tardio obtidas podem modificar significativamente as curvas atuariais prévias. No último ano deste estudo, ocorreu um óbito hospitalar adicional no Grupo $A$, o que já representa, para estes pacientes, "sobrevida imediata" de $87,5 \%$, inferior aos $90 \%$ observados aos 60 meses, no trabalho do "Annals". A partir deste percentual, a aplicação cumulativa dos demais dados obtidos no seguimento tardio explicam as diferenças entre os dois trabalhos. 